\title{
INVESTIGACION DE MERCADO EN LA FACULTAD DE INGENIERÍA INDUSTRIAL
}

Ing. Rolando Carrión Muñoz

\section{Resumen}

El presente artículo muestra la aplicación de la investigación de mercados en el Maquicentro de la Facultad de Ingeniería Industrial de la UNMSM.

Abstract

This article shows the application of the investigation of markets in the Maquicentro of College of Industrial Engineering of the UNMSM.

\section{Introducción:}

\section{Las Funciones de los Mercados}

Al igual que la sociedad en su conjunto, también las empresas y en nuestro caso el Maquicentro de la Facultad de Ingenieria Industrial debe resolver permanentemente tres problemas fundamentales:

1) Qué bienes y servicios debe producir y en qué cantidades,

2) Para quién debe producirlos y

3) Cómo debe producirlos

La solución de esos tres problemas básicos obliga a los administradores de dicho centro de producción a tomar, diariamente, una serie de decisiones, las mismas que pueden tomar basándose únicamente en su propia experiencia y conocimientos o con la ayuda de otros expertos y de las investigaciones del mercado.

Las investigaciones de mercados, como las investigaciones en cualquier otro campo, deben ser realizadas por expertos, por profesionales conocedores tanto del área dentro del cual se realizará la investigación, como de las técnicas de investigación y análisis aplicables en cada caso.

\section{El Estudiante Investigador}

Tratándose del estudiante de investigación de mercados, los conocimientos de las técnicas de investigación están dados por el aprendizaje de los temas de la propia asignatura; en cuanto al conocimiento de la actividad productiva que interesa, en la mayor parte de las veces no la conoce en detalle y, casi en la generalidad de los casos, no ha podido especializarse aún en ella, luego, debe suplir estas deficiencias antes de ejecutar una investigación de mercados formal.

\section{La Investigación en la Facultad}

\section{La Investigación Exploratoria}

Presentamos a continuación en forma breve los resultados de las investigaciones realizadas en el curso de Investigación y Análisis de Mercados durante el Semestre Académico 1999-I, ellas ofrecen información que permitirá reducir la incertidumbre de los responsables al tomar las decisiones relacionadas con la producción y comercialización de los bienes y servicios que ofrece la Facultad

Los principales medios que han permitido a los estudiantes un rápido conocimiento de la actividad productiva dentro de la cual han realizado sus investigaciones son tres:

1. La lectura de todo tipo de documentos: escritos, grabados o transmitidos por el INTERNET, relacionadas con la actividad de interés, a los que han tenido acceso.

2. La obtención de datos históricos y actuales de casos similares, con la finalidad de determinar semejanzas, diferencias y particularidades entre ellos y el que motiva el estudio.

3. Finalmente, la entrevista con expertos, de los han obtenido información no sólo acerca de las principales fortalezas y debilidades más frecuentes en determinado tipo de industria, sino también acerca de la manera cómo es posible superar las dificultades y, aprovechar las oportunidades que se pueden presentar. A falta de expertos algunos han tenido que recurrir a la alternativa de cambiar impresiones y opiniones con personas muy observadoras e imaginativas, lo que les ha permitido realizar una gimnasia mental que los ha habilitado a captar mejor las diferentes facetas de la actividad que muy rápidamente han llegado a conocer, por sus propios medios, con el mayor detalle posible, a pesar de la brevedad del tiempo que dura un semestre de clases, en cuyo lapso, además, han tenido que realizar otros estudios.

\section{La Investigación Concluyente}

Si bien, los problemas de decisión formulados por los responsables de la gestión de los talleres del Maquicentro parecen no tener relación con los que 
hemos mencionado al principio, un breve análisis nos permite relacionarlos con mucha facilidad.

A continuación una descripción breve de los trabajos realizados por los alumnos:

1. Investigación y Análisis de Mercado para el Lanzamiento de Nuevos Productos de la Panaderia de la Facultad. Paúl Peter Chinchón Morrillo, Elliot Jacinto Rios, Eloy Huamantupa Riscco, Muller Martino Landacay y Elmer Monsalve Padilla.

La principal conclusión de este estudio, sobre la base de una muestra aleatoria de consumidores es que el $80 \%$ está dispuesto a comprar panes elaborados con harinas alternativas a la harina de trigo, este conocimiento permite realizar experimentos a bajo costo.

Otras conclusiones relacionadas con la compra de pan en la panadería de la Facultad son:

- Al $92 \%$ le interesa conocer el valor nutritivo del pan.

- El $74 \%$ gasta entre S/.0.60 y S/.1.00.

- El $80 \%$ realiza sus compras fuera de las horas de comida.

- El $21 \%$ compra de 5 a 6 veces por semana, el $40 \%$ de 2 a 4 veces y el $39 \%$ una vez por semana.

2. Investigación para el Maquicentro de la Facultad de Ingeniería Industrial Sección Calzado. Liliam Manrique Gómez, Lucy Mesía Pizarro y Wilman Ñahui Ccaccya.

Las conclusiones más importantes de este estudio realizado sobre una muestra de clientes y no clientes, con relación a las mayores preferencias son:

- Tipo de calzado para la próxima estación: el sport.

- Material: cuero.

- Aplicación: hebilla.

3. Análisis de Mercado del Centro de Extensión Universitaria y Proyección Social (Área de Informática) CEUPSI. Edwin Chuquitaype Cayo, Pedro Rivera Mejia, Jorge Chávez Acosta y Henry León Masgo.

Este estudio realizado sobre una muestra aleatoria de alumnos mostró principalmente que:

- En general la imagen del CEUPSI es muy favorable

- Se debe mejorar la presentación de las separatas

- Se debe incrementar las horas de práctica

4. Estudio de la Aceptabilidad de los Calzados elaborados en el Maquicentro de la FII de la UNMSM. Roberto Merino Ascarrunz y Sandro Vásquez Paz.
En este estudio interesaba determinar, por un lado las caracteristicas que influyen en la decisión de compra, de otro lado los motivos por los cuales volvería a comprar o no los actuales clientes.

- Respecto de lo primero se determinó que el diseño, el acabado, el precio y la comodidad del calzado son las características que influyen en la decisión de compra. 23.5\% de los que compraron lo hicieron por el precio, $26.5 \%$ por el diseño, $41 \%$ por las facilidades de pago y el $9 \%$ por otros motivos.

- Respecto de lo segundo, los motivos más importantes para volver a comprar son el precio y las facilidades de pago, para no comprar la corta duración y el acabado.

5. Maquicentro Confecciones, José Luis Cabello Barrenachea, Claudio Caballero Castillo, Marilú Matsutahara Higa, Anthony Jesús Salinas Cavero, Luis Samuel Villamón Cifuentes.

Mediante este estudio de mercado se ha determinado en primer lugar la imagen que se ha formado el estudiante de San Marcos, del Maquicentro de la Facultad; en segundo lugar, cuál es el diseño preferido del logotipo que debe ir bordado en las camisas e impreso en las bolsas de dicha prenda.

Los alumnos que han realizado las investigaciones que hemos descrito brevemente son ahora mejores conocedores de sendas actividades productivas, de lo que eran al inicio del semestre académico y, muy aparte de las conclusiones a que han llegado en su trabajo de investigación de mercados, sus conocimientos y opiniones pueden ser aprovechados por quienes deben tomar decisiones en el Maquicentro de la Facultad. Ellos están dispuestos a exponer ante las autoridades de la Universidad y contribuir de ese modo con el desarrollo de su alma mater.

\section{Conclusión Final}

Estamos convencidos de que tanto o más importantes que los conocimientos adquiridos por los estudiantes en las aulas, son los que ellos adquieren por su propia cuenta y esfuerzo, porque de este modo se habitúan en conseguir la información que necesitan, perfeccionándose en forma permanente.

Además conviene hacer hincapié en la necesidad que tienen los alumnos en completar sus conocimientos de Investigación de Mercados con los de Mercadotecnia, disciplina que les ofrecerá conocimientos totalmente diferentes que los habilitará para manejar adecuadamente las variables de la mezcla de mercadeo y las técnicas de comercialización, pudiendo asi entender, formular aplicar y conducir planes de marketing. 